\title{
TRABAJO EN EQUIPO: DE LA TEORÍA A LA CULTURA INSTITUCIONAL
}

María Ema Pintos Artigas*
Fernando Martínez Sagasta**

\section{Introducción}

Quienes realizamos este estudio, Coordinadores Adscriptos en el área de $5^{\circ}$ y $6^{\circ}$ año de bachillerato de una institución educativa privada, trabajamos desde hace veinte años en la formación, seguimiento y acompañamiento personal y grupal de sus estudiantes. Nuestras experiencias en el ámbito de la misma, nos permite percibir que los distintos educadores que integramos los diferentes campos disciplinares, cuando nos reunimos para trabajar en equipo, en reiteradas oportunidades lo hacemos en grupos separados, especialmente con relación a nuestras disciplinas, o, adoptando formas limitadas, en el sentido de que los educadores no ejercemos la enseñanza conjunta dentro del aula. Se trata de un trabajo en equipo que se concentra en lo inmediato, en el corto plazo y excluye los problemas de planificación del plazo más largo, o de una forma impuesta o más dirigible, que es buena para cuando se inicia en la modalidad de trabajo en equipo, pero que no es deseable se extienda en el tiempo, -lo que constatamos ocurre en ocasiones-. Frente a estas percepciones deseamos indagar cómo estamos interactuando los educadores en los diferentes equipos de trabajo.

Consideramos al trabajo en equipo como enriquecedor de los procesos educativos y le otorgamos gran importancia a su proyección en el tiempo, por esto pensamos que nuestro aporte puede colaborar en que se llegue a esta toma de conciencia, e ir poco a poco, transformando la teorización del tema en una verdadera cultura de trabajo en equipo. Nuestro propósito, pues, es indagar si el trabajo en equipo es favorecido o no, por la interacción entre los educadores. Para orientar nuestro estudio nos planteamos la siguiente interrogante: ¿la interacción entre los educadores favorece o no, el trabajo en equipo en $5^{\circ}$ y $6^{\circ}$ año de bachillerato de la institución en que trabajamos? Abordando aspectos que surgen de esta pregunta y que sirven como aproximación, la desagregamos en: ¿quién determina los equipos?, ¿para qué funcionan?, ¿cómo están funcionando?, ¿qué tipo de equipos trabajan?, ¿qué logros alcanzan estos equipos?.

\section{Marco teórico}

Sin duda el trabajo en equipo no es sencillo, conlleva dificultades y deficiencias que son muy frecuentes. Fullan y Hargreaves (1999), describen tres formas de trabajo en equipo que nos permiten visualizar estas realidades y ejemplifican muy bien sus carencias. 1) La "balcanización", en la que el trabajo se da en grupos particulares, se unen quienes trabajan más estrechamente; en ocasiones estos grupos sustentan puntos de vista diferentes respecto del aprendizaje, las formas de enseñanza, la disciplina, el currículum. Se integran a estos grupos, tanto educadores conservadores como innovadores. La balcanización puede traer falta de comunicación, indiferencia, rivalidad y el que los educadores transiten por senderos distintos y a veces opuestos. 2) El trabajo en equipo "fácil", descripto como aquél que adopta formas limitadas en lugar de amplias. Los principales componentes del aislamiento permanecen indemnes, es decir, los educadores planifican juntos pero no comparten en el aula, perdiendo así la riqueza de la mutua observación, la investigación y el consejo acerca de sus prácticas. No se trabaja sobre el modo de enseñar, no tiene en cuenta los principios de una reflexión, el cuestionamiento, la indagación, el diálogo y la misma crítica; el trabajo en equipo no debe limitarse a la cercanía, a la afinidad, y debe estar dispuesto a enfrentar discusiones, desacuerdos y conflictos de distinta índole para 
poder alcanzar una cooperación eficaz. 3) El trabajo en equipo "artificial", o modalidad más controlada por las jerarquías, en la cual éstas pueden regular o dominar; se caracteriza por la implementación de procedimientos formales, específicos, tendientes a la planificación conjunta entre los educadores, dejando de lado metas que no se pueden predecir con antelación. Esta modalidad tiene posibilidades negativas y positivas según cómo y cuándo se ponga en práctica. Puede quedar reducida a un sustituto administrativo de las culturas del trabajo en equipo, disminuyendo la motivación en cuanto a la cooperación entre pares, o puede ser el preámbulo de relaciones cooperativas más duraderas y eficaces entre los educadores.

Encontramos muy adecuado y relevante lo que estos autores expresan: "...las culturas del trabajo en equipo son muy complejas. No se pueden crear de la noche a la mañana. Muchas formas de trabajo en equipo son superficiales, parciales e incluso contraproducentes. No es posible tener culturas fuertes de trabajo en equipo sin un vigoroso desarrollo del individuo. Debemos evitar aplastar la individualidad en el impulso de eliminar el individualismo. Al mismo tiempo, no se debería dejar completamente solos a los maestros ni ellos mismos se deberían desasistir. El estímulo y la presión de un profesionalismo interactivo instituido servirán de fuente continua de nuevas ideas y sustentos, y de una forma de responsabilidad más adecuada a la profesión de enseñar, con sus elevadas exigencias y necesaria sabiduría"4.

Compartimos el concepto de cultura con estos autores como las creencias y expectativas orientadoras de cada escuela, haciendo énfasis en las relaciones entre las personas. En síntesis, "la manera como hacemos las cosas y nos relacionamos los unos con los otros"5. Ampliando estos conceptos también nos adherimos a las ideas de Marchesi y Martín (1998) en cuanto a que la cultura es el conjunto de las creencias y convicciones que tenemos los educadores y la comunidad educativa en relación con la enseñanza, el aprendizaje y el funcionamiento. Incluye las relaciones que se establecen, las normas que afectan a la comunidad y a los procesos de enseñanza y aprendizaje, la comunicación y la colaboración entre los educadores.

No debemos considerar la cultura sin tener en cuenta la estructura del centro, entendiendo como estructura al sistema organizativo, la toma de decisiones, el tipo de participación y los roles de los distintos actores. Existe una interdependencia entre ambas. Las estructuras generan las culturas, los cambios en la cultura pueden llegar a modificar la estructura que existe. Por otra parte, el cambio en la cultura si no afecta a la estructura organizativa, puede quedarse sin sustento para proporcionarle validez y fortaleza. "En consecuencia, en algunos casos, no es posible establecer unas culturas escolares productivas sin que, antes, se efectúen cambios en las estructuras escolares que aumenten las oportunidades para unas relaciones de trabajo significativas y un apoyo colegial entre los profesores"6. Los cambios culturales presentan el objetivo de apoyar la labor de los distintos equipos de trabajo y favorecen la comunicación, la colaboración y el apoyo mutuo. Es evidente que cambios hacia estilos de mayor colaboración y flexibilidad organizativa ayudarán a mejorar las prácticas de enseñanza en el aula, lo que mejorará la calidad de la enseñanza.

Rosenholtz (1989) hace una diferenciación verdaderamente significativa en relación con las culturas escolares. Escuelas "en movimiento" o de aprendizaje enriquecido, y las escuelas "atascadas" o de aprendizaje empobrecido. Asimila bajo rendimiento, con docentes que por lo general trabajan solos, sin solicitar ayuda, "atascados". En las escuelas "en movimiento", la realidad es bien distinta, los docentes trabajan en equipo, reconocen lo difícil de la enseñanza, la necesidad de recibir ayuda y la solicitan sin que esto sea signo de incompetencia. La mejora de la enseñanza es más colectiva que individual. El análisis, la evaluación y la experimentación en compañía de nuestros pares, son condiciones bajo las cuales los educadores mejoramos. 
Otro aspecto que queremos destacar y que favorece el trabajo en equipo son las cualidades, actitudes y conductas que marcan las relaciones personales en todo momento, tales como la asistencia, apoyo, confianza, sinceridad. Eso lleva a valorar tanto a las personas en lo individual cuanto en lo referido a los grupos a los que pertenecen. Todo lo anterior ayuda a tolerar las discrepancias, ya que las culturas de trabajo en equipo tienen una buena base sólida de seguridad apoyada en las relaciones personales. Las culturas de trabajo en equipo respetan y tienen en cuenta al educador como persona y reconocen su intención. Por otra parte debemos de tener en cuenta que una buena sociabilidad y un buen clima, no aseguran una gran eficacia; estamos completamente de acuerdo con la idea de que "cuando se trata de desarrollar culturas de trabajo en equipo no hay que confundir felicidad con excelencia"7.

En las escuelas de calidad los educadores somos valorados en nuestro esfuerzo individual y en la participación en equipos de trabajo. Estas escuelas tienen un proyecto en común, están abiertas a la comunidad, sus integrantes buscan objetivos en común, son flexibles en la enseñanza y el aprendizaje de los alumnos, y son lideradas dinámicamente. Es nuestra convicción que las instituciones educativas deben formular estrategias, crear espacios, alentar disponibilidades, aunar esfuerzos individuales, en la búsqueda de la transformación del trabajo en equipo en verdadera cultura institucional, lo que sin duda beneficiará la interacción entre los integrantes de la comunidad educativa. Entendemos que los conceptos anteriormente expresados, vinculados al trabajo en equipo, tales como participación, comunicación, planificación, estilos de gestión, modalidades y formas de trabajo, estructura, cultura, profesionalismo educativo, relaciones interpersonales, cooperación, individualidad e individualismo, escuelas "en movimiento" y "atascadas", escuelas de calidad y otros, nos resultan un insumo sumamente útil para realizar nuestro análisis con el interés de contestar las interrogantes que nos planteamos respecto de la indagación propuesta.

\section{Metodología}

Si bien en nuestro estudio hacemos una combinación de los atributos naturalistas y holísticos del paradigma cualitativo con los atributos comprobatorios del cuantitativo, afirmamos que nuestra investigación presenta un enfoque fundamentalmente cualitativo dada la naturaleza del objeto de estudio. "La frase metodología cualitativa se refiere en su más amplio sentido a la investigación que produce datos descriptivos: las propias palabras de las personas, habladas o escritas, y la conducta observable". La investigación cualitativa nos permite estudiar el contexto natural en donde las personas sienten, perciben, actúan, buscando comprender sus diferentes concepciones acerca de los fenómenos, a partir de los datos obtenidos y su posterior análisis.

\subsection{Campo de investigación}

Esta investigación la llevamos a cabo en el área de $5^{\circ}$ y $6^{\circ}$ año de bachillerato de la institución educativa que ya describimos. El universo en estudio está constituido por treinta y ocho educadores. Elegidos con un criterio de corte muestral intencional de tres o más integrantes por equipo, para lograr una medición y acercamiento más significativos al buen funcionamiento y organización de los mismos, consideramos siete equipos en total: cuatro equipos de tres educadores, uno de cuatro educadores y otro de cinco, que funcionan en relación con sus disciplinas; y un equipo de ocho, llamado Interdisciplinario, integrado por la Directora, la Coordinadora de área, cuatro Coordinadores Adscriptos, el Coordinador de Pastoral y la Psicóloga. Este equipo, creemos sea asimilable a lo que Fernández expresa como "grupos intersticiales"9: aquellos que se reúnen para tareas de conexión y articulación. Los nueve restantes no integran equipos de tres o más miembros por la conformación de las asignaturas, por lo que no serán incluidos en la muestra. A continuación presentamos la matriz metodológica que detalla la organización conceptual del proceso de investigación: 


\begin{tabular}{|l|l|l|l|}
\hline Dimensión & Variable & \multicolumn{1}{|c|}{ Indicador } & Instrumento \\
\hline \multirow{4}{*}{$\begin{array}{c}\text { Pedagógico } \\
\text { Didáctica }\end{array}$} & Contenidos & Planificación previa & Entrevista \\
\cline { 2 - 4 } & Propuesta metodológica & Modalidad de propuesta & Entrevista \\
\cline { 2 - 4 } & Evaluación & Criterios de evaluación & Entrevista \\
\hline & Liderazgo & Estilo de liderazgo & Encuesta \\
\cline { 2 - 4 } & Coordinación & Niveles de coordinación & Encuesta \\
\cline { 2 - 4 } & Logros & $\begin{array}{l}\text { Grado de satisfacción con } \\
\text { respecto a los logros alcanzados }\end{array}$ & Encuesta \\
\cline { 2 - 4 } & Toma de decisiones & Carácter de las decisiones & Entrevista \\
\cline { 2 - 4 } & Comunicación & Modalidades de comunicación & Entrevista \\
\cline { 2 - 4 } & Participación & Finalidades de la participación & Entrevista \\
\cline { 2 - 4 } & Conflictos & Ubicación de los actores & Entrevista \\
\hline
\end{tabular}

Las principales dimensiones tomadas para el análisis serán dos: la pedagógico didáctica y la organizacional. La dimensión pedagógico didáctica hace a la institución educativa, es por esto que existe: le da especificidad, "constituyendo a las actividades de enseñanza y aprendizaje en su eje estructurante, actividad sustantiva y específica" ${ }^{10}$, entendiendo lo pedagógico en su más amplio sentido, es decir vinculado con todo lo educativo. La dimensión organizacional hace a la dinámica en cuanto a los roles, las funciones relacionadas con éstos, y temas conexos como las variables correspondientes a esta dimensión que figuran en la matriz. Lo organizacional está al servicio de lo pedagógico didáctico. Indagamos cómo interactúan los educadores en los equipos de trabajo referidos a estas dos dimensiones.

\subsection{Herramientas}

Las herramientas que utilizamos para la colecta de datos han sido la encuesta y la entrevista semiestructurada. La encuesta, sumamente útil como forma de aprehender un determinado mundo de percepciones que hallamos en el universo en su totalidad. Entendemos que todos los educadores del bachillerato integren o no equipos de trabajo, tienen una idea formada con respecto a tres variables consideradas macro, como el liderazgo, la coordinación y los logros; las tres pertenecientes a la dimensión organizacional. La misma consta de preguntas y afirmaciones, pasibles de ser cuantificadas en sus resultados. Aplicamos la totalidad de las encuestas, treinta y ocho, y recibimos contestadas treinta y cinco, lo que representa un noventa y dos por ciento de retorno. Y la entrevista semiestructurada es ciertamente válida para extraer información de una persona determinada, por eso estará teñida por su biografía, es decir, sus propias vivencias, no es una información aséptica y de ahí su real valor. Parafraseando a Blanchet, podemos decir que "la subjetividad del producto informativo generado es una propiedad de las entrevistas"11. Planificamos nueve entrevistas, una a un integrante de cada equipo, elegido al azar; y las otras dos a informantes claves o calificados, siendo estos la Directora y el Coordinador de Pastoral. Ambos, por los roles que desempeñan tienen un conocimiento global y no solo parcial de los educadores que trabajamos en bachillerato y de los respectivos equipos de trabajo. Elaboramos las pautas para ordenar dichas entrevistas, siendo la referencia las tres variables consideradas en la matriz, de la dimensión pedagógico didáctica y las restantes de la dimensión organizacional que no fueron consultadas en la encuesta. Estas siete variables las consideramos específicas y muy significativas para el estudio de equipos de trabajo a nivel educativo. Realizamos las entrevistas uno u otro investigador indistintamente, todos los entrevistados aceptaron de buen grado y se extendieron en sus respuestas, lo que permitió una gran riqueza en la información que revelaron. Las mismas fueron grabadas, previo consentimiento, y 
tuvieron una duración aproximada de cuarenta minutos cada una. Luego fueron desgrabadas y analizadas en forma independiente, por cada uno de nosotros. Más tarde hicimos una puesta en común y análisis en conjunto. Las dos herramientas fueron testeadas previamente a su puesta en marcha, con el propósito de verificar su pertinencia para la recolección de la información buscada.

La triangulación que llevamos a cabo con los datos e información obtenidos mediante ambos instrumentos, nos permitió visualizar su complementariedad, aumentando el valor de la información recabada. El estudio conjunto disminuyó las limitaciones que hubiera tenido su análisis por separado.

\section{Análisis}

Lo realizamos retomando las dimensiones pedagógico didáctica y organizacional y sus respectivas variables, constituyentes de la matriz metodológica antes descripta. Su organización fue de lo general a lo particular.

Los datos cuantitativos obtenidos muestran, en un análisis primario, la predominancia del apoyo de la Dirección al trabajo en equipo, con escasa influencia en su labor y pocas orientaciones pedagógico didácticas por parte de la misma. Se refleja esto también en las prioridades de una Coordinación que jerarquiza aspectos organizacionales, positivamente conceptuada y facilitadora de las interacciones entre los educadores. Indican que se sienten medio a poco conformes en relación a los logros conseguidos y que estos, a veces, son tomados como insumos para el futuro, con una tendencia a ser tenidos en cuenta en forma más frecuente.

Complementando el análisis de la encuesta con las entrevistas, encontramos que existe una tendencia positiva $(42,9 \%$ a $88,6 \%)$ en cuanto al apoyo y el estímulo del trabajo en equipo por parte de la Dirección, y si bien se afirma mayoritariamente (siete de nueve entrevistados) que es llevado a cabo, existen diferentes matices en cuanto a la conceptualización del mismo. Por otra parte, aquellos educadores que por su rol tienen una visión más global del bachillerato, Directora y Coordinadores, conocen los distintos equipos de trabajo, mientras que los demás entrevistados (seis de nueve) conocen solamente de los que tienen relación directa, y mientras que de los otros, su conocimiento es difuso o poco claro, a excepción del equipo interdisciplinario.

Este equipo, habiendo sido creado por la Dirección General, se reúne sistemáticamente hace nueve años, realizando tareas de planificación, articulación, conexión y funcionamiento general, manteniendo un contacto permanente con el resto de los educadores. En el conjunto de los entrevistados y encuestados prevalece un conocimiento parcial respecto de los demás equipos, que son aquellos que corresponden a seis disciplinas diferentes, integrados por los respectivos docentes, en forma voluntaria y espontánea, orientados al desarrollo sin circunscribirse a un tiempo o espacio determinados, siendo esporádicamente reunidos por la Dirección para temas puntuales.

En cuanto a la participación, hay un estímulo por parte de la Dirección para el trabajo en equipo. Asociándose a esto, la Coordinación es considerada eficaz ( $45,7 \%$ a $80 \%$ ) y se piensa que favorece en la interacción entre los miembros de los equipos, reflejándose esto en los resultados de la encuesta $(42,9 \%$ a $88,55 \%)$. En cuanto a la finalidad de participar en estos equipos, surge un predominio de aspectos estructurales relacionados con lo organizacional. Los entrevistados expresaron: "el coordinar, dar distintos puntos de vista", "compartir las experiencias de otros", "puesta en común", "escucharse", "apoyo afectivo", "los aportes de cada uno, la crítica al otro inclusive en el error", "no sentirse solos y poder superar inseguridades". Manifestaron en forma escasa, enfoques pedagógicos y didácticos, "afinar programas", "proyecto educativo en común".

Queremos hacer notar la coincidencia que existe entre la finalidad de la participación que manifestaron los entrevistados con lo que surge de la encuesta referido a los aspectos más jerarquizados por la Coordinación ( $60 \%$ a $85,7 \%$ ), que a su vez los educadores consideran de importantes a muy importantes dentro de la institución, el predominio de enfoques organizacionales. 
Existe poca o escasa planificación previa en cuanto a los contenidos a desarrollar (seis en nueve entrevistados lo afirmaron). Diversas razones fueron dadas por la mayoría de los entrevistados, tales como: "tendencia al aislamiento, falta de coordinación en las asignaturas, no estar institucionalizado, no estar organizado, falta de hábito, no hay cultura", "ausencia de alguien que dirija, la existencia de cotos o feudos inexpugnables", "los años compartidos hacen que cada uno sepa lo que le sirve al otro docente", "falta afinar la parte metodológica de la planificación". Una minoría (tres en siete) de los equipos, sí planifica previamente.

En la modalidad de trabajo a implementar, tampoco hay acuerdos, a excepción de dos de los entrevistados que sí dijeron realizar una propuesta metodológica en conjunto en sus respectivos equipos. Se mencionó que "no existen pautas metodológicas", "que debería haber acuerdos sobre bases reales y buscar la mejor metodología para el alumno", "tiene que haber alguien indicado por Dirección para determinadas cosas, alguien que sepa de la materia", "tenemos métodos tan dispares que acercar las posiciones es muy difícil", se repitió "que existen esfuerzos aislados que quedan en el camino", "deberían darse acuerdos previos formales y sistematizados", incluso uno de los entrevistados dijo: "no, cada uno tiene su forma".

Si bien la mayoría (seis en nueve) de los entrevistados respondió que los criterios de evaluación no son elaborados en conjunto en el transcurso del año, sí hay acuerdos en aspectos puntuales como por ejemplo en el período de exámenes. Queremos rescatar que los entrevistados A y E y H contestaron que se ponen de acuerdo en sus respectivos equipos en el correr del año. Concretamente el entrevistado A dijo "sí, son discutidos abiertamente", el entrevistado E señaló que "la evaluación forma parte de la planificación", y el entrevistado H expresó "hemos discutido, yo he traído aportes y a lo largo del tiempo llegamos a acuerdos". En cuanto a la toma de decisiones, los entrevistados manifestaron que tanto los equipos como sus respectivos integrantes tienen posibilidades de tomarlas en aspectos relevantes, ya sea por ejemplo en "los procesos de la Pastoral del Colegio y grupos juveniles", "en la búsqueda de un método de trabajo en común", "en la elección de los temas más importantes para los cursos", "en las distintas formas de evaluar", "el poder resolver en relación a disciplina, en la imposición de límites". Se valora la libertad y autonomía que existen.

Consultados sobre la comunicación, el total de los entrevistados expresó que a nivel personal es de buena a muy buena, destacando lo positivo de las relaciones interpersonales. En cuanto al trabajo en equipo manifestaron que "no hay estilo de comunicación", "que es informal", "que no hay canales formales de comunicación", "no hay una comunicación académica, es esporádica y no fructífera", "no está asegurada porque gira en torno a las personas", contestó, que tanto a nivel personal como de trabajo en equipo, "se buscan los mecanismos más adecuados y eficaces" manteniendo un contacto sistemático.

En el caso de surgir un conflicto, como postura personal todos los entrevistados coincidieron en que intentan solucionarlo, no dejándolo de lado, ya sea mediando, haciéndolo explícito, dialogando respetuosamente. En cuanto a la posición del resto del equipo, surgieron diferentes respuestas: "algunos lo eluden, otros lo enfrentan", "hay quienes lo evitan por no separar lo profesional de lo personal", "restándole efectividad a la tarea", expresó que "el conflicto es una forma de crecimiento del equipo y de los integrantes del mismo". El trabajo en equipo supone, entre otras cosas, el ansia y la búsqueda consciente e inconsciente de logros, tanto sean personales como grupales. Debemos destacar que predomina $(45,7 \%$ a $82,8 \%$ ) un sentimiento de insatisfacción en cuanto a logros alcanzados, aunque en su mayoría ( $48,6 \%$ a $88,6 \%$ ) están dentro de los objetivos propuestos. Se piensa que a veces $(57,15 \%)$ se ven cumplidas las expectativas personales de los educadores encuestados, al igual que en ocasiones son tenidos en cuenta para posteriores trabajos $(51,45 \%)$.

Para una mejor observación de la información analizada, presentamos la siguiente tabla, en donde categorizamos algunas características de las interacciones que, de acuerdo con la percepción de los educadores son "favorables" y "no favorables" para el trabajo en equipo. 


\section{Características de las interacciones entre los educadores}

\begin{tabular}{|c|c|}
\hline Características que favorecen & Características que no favorecen \\
\hline $\begin{array}{l}\text { Apoyo y estímulo de la Dirección al trabajo } \\
\text { en equipo y a la participación. }\end{array}$ & $\begin{array}{l}\text { Escasas orientaciones pedagógico didácticas } \\
\text { por parte de la Dirección. }\end{array}$ \\
\hline No influencia de la Dirección en la tarea. & Poca planificación previa en cuanto a contenidos. \\
\hline $\begin{array}{l}\text { Coordinación eficaz y facilitadora de } \\
\text { interacciones entre los educadores }\end{array}$ & $\begin{array}{l}\text { Minoría de acuerdos previos en relación con la } \\
\text { metodología de trabajo. }\end{array}$ \\
\hline $\begin{array}{l}\text { Existencia de acuerdos en la evaluación } \\
\text { para temas puntuales como por ejemplo } \\
\text { exámenes. }\end{array}$ & $\begin{array}{l}\text { Los criterios de evaluación no son elaborados } \\
\text { en conjunto en la mayoría de los equipos, } \\
\text { salvo para temas puntuales. }\end{array}$ \\
\hline $\begin{array}{l}\text { Posibilidad de toma de decisiones en } \\
\text { aspectos relevantes. }\end{array}$ & $\begin{array}{l}\text { El trabajo en equipo es poco evaluado por la } \\
\text { Dirección. }\end{array}$ \\
\hline Libertad y autonomía en el hacer. & $\begin{array}{l}\text { Estilo de comunicación informal, dependiendo } \\
\text { de las personas. }\end{array}$ \\
\hline Relaciones interpersonales positivas. & $\begin{array}{l}\text { En ocasiones, en los conflictos, no se separa } \\
\text { lo personal de lo profesional, lo que impide } \\
\text { superarlos. }\end{array}$ \\
\hline $\begin{array}{l}\text { Solución de conflictos en base al diálogo y } \\
\text { el respeto. }\end{array}$ & Tendencia al aislamiento. \\
\hline $\begin{array}{l}\text { Los logros que se alcanzan son tomados } \\
\text { como insumos útiles. }\end{array}$ & $\begin{array}{l}\text { Sentimiento de insatisfacción en cuanto a los } \\
\text { logros alcanzados. }\end{array}$ \\
\hline
\end{tabular}

\section{Conclusiones}

Si bien en la currícula del bachillerato no está presente la figura de la coordinación por asignaturas, como existe en primer ciclo, tampoco está instaurado el trabajo en equipo, tanto sea en torno a las disciplinas como entre ellas. Cabe destacar que en nuestra institución se ha iniciado un camino, aunque informal y poco sistematizado, existiendo un apoyo y estímulo de la Dirección en relación con el trabajo en equipo, ambos percibidos por los educadores en su mayoría. Contribuye a lo anterior que la no influencia de la Dirección en los equipos de trabajo es tomada como algo positivo, así como también la Coordinación existente es considerada eficaz.

Valoramos enormemente el sentido de autocrítica que demuestran los educadores, revelando sin reservas las falencias o insuficiencias que les son propias, en relación con aspectos pedagógico didácticos, tales como la falta de planificación previa, el no tener acuerdos en relación con metodología y evaluación, y en algunos aspectos organizacionales como la comunicación.

Están presentes en todas las entrevistas las creencias y convicciones de los educadores referidas a lo favorable y positivo de la modalidad del trabajo en equipo, a pesar de que no se dé, o que sea insuficiente, o adquiera en oportunidades facetas no deseables como la "balcanización", el trabajo en equipo "fácil" o "artificial" descriptos por Fullan y Hargreaves (1999). No tenemos aún criterios comunes en relación con la enseñanza, el aprendizaje y el funcionamiento, lo que lleva a que exista una tendencia al aislamiento. 
Comprobamos, sin lugar a dudas que las culturas de trabajo en equipo son muy complejas y que si bien se vislumbran atisbos de ellas, pensamos que deberíamos como institución cambiar o intensificar aspectos instrumentales que modifiquen la estructura. Sugerimos lo anterior por convencimiento y a la luz de lo expresado por Marchesi y Martín (1998), en relación con cambios hacia culturas escolares productivas que requieren de cambios estructurales que tiendan a relaciones de trabajo significativas y a un apoyo colegial entre los profesores. Es de destacar la libertad y autonomía mencionadas por los entrevistados con respecto de la toma de decisiones, pilar fundamental que favorece el trabajo en equipo. Habla de una flexibilidad organizativa, que junto a estilos de mayor colaboración seguramente podrán contribuir a la mejora de la enseñanza.

Pensamos que este estudio nos revela la convicción que tienen los educadores respecto de las bondades del trabajo en equipo, del real alcance que se puede lograr cuando éste es relevante y significativo; y que la mirada reflexiva que posibilita el descubrir carencias, errores, así como también puntos fuertes no hace más que ayudar a intentar potenciar capacidades tanto personales como de equipo, llevando a la institución a estar "en movimiento".

\section{Discusión}

Entendemos que el propósito de la investigación se cumplió, permitiéndonos dar respuesta tanto a la pregunta orientadora, como a las preguntas en que fue desagregada. De acuerdo con lo planificado, y recorriendo el proceso de investigación y su análisis, surge quién determina los equipos, para qué y cómo están funcionando, los tipos de equipos que trabajan y el alcance de sus logros. Todo esto ayudó a elaborar las conclusiones anteriormente descriptas.

\subsection{Limitaciones de este trabajo de investigación}

Como toda investigación de corte cualitativo, no es generalizable y por ende sus resultados deben ser relativizados, así como también las percepciones de los educadores, por ser tales, no son transferibles. Por otra parte nueve educadores de los treinta y ocho, no integran equipos de trabajo de tres o más miembros, lo que limita nuestro estudio, incidiendo en las interpretaciones y conclusiones ya que no abarca el total del universo institucional.

\subsection{Perspectivas}

El largo camino, Trabajo en equipo: de la teoría a la cultura institucional, tiene un comienzo y unos primeros pasos que ya han sido dados; sin duda debemos seguir avanzando. No hay soluciones mágicas, ni certezas perdurables, lo cierto es que el trabajo de los educadores y sus elevadas exigencias están cambiando al igual que el mundo que nos circunda; si todos y juntos no nos ponemos en situación decidida de afrontar estos cambios, que tienen una voz cada vez más fuerte y resonante, nos quedaremos detenidos en el tiempo, paralizados por la incertidumbre y culpabilizados por tener en nuestras conciencias la carga de la ausencia o la limitación de respuestas ante demandas cada día más complejas y plurales.

En la teoría, así como en la mayoría de los discursos registrados, se visualizan muchas de las soluciones a nuestros errores o carencias, tanto en lo que nos es personal como en lo grupal, así como también lo referido a lo institucional. Tenemos un valor cada vez menos corriente; una buena autocrítica, y contamos también con una institución que muestra la voluntad política de ir acompañando y alentando, $\mathrm{y}$, en ocasiones, motivando estos cambios. 
Entonces, nos preguntamos, a sabiendas de que las culturas de trabajo en equipo y colaboración son muy complejas y difíciles de ser alcanzadas, ¿qué nos está limitando, qué obstaculizando?, o mejor aún ¿a qué estamos dispuestos, qué ofrecemos, qué intentaríamos modificar de nuestros comportamientos personales y profesionales?, ¿qué necesitamos como aportes de la Institución, qué nos puede brindar ella, en qué y cómo puede ir transformándose?, ¿en qué podemos todos fortalecernos?; sin olvidar nuestro carisma, la riqueza histórica, nuestras formas y maneras de hacer las cosas y relacionarnos entre nosotros y con lo que nos rodea, enriqueciendo nuestros valores de antaño con los que vamos incorporando por éticos, necesarios y comunitarios.

Tal vez el camino sea un reto, el reto de la reestructuración en la educación. Nos preguntamos: ¿cómo hacerlo viable?, ¿qué tenemos que hacer para que lo que queremos que pase, ocurra realmente?

\subsection{Propuestas a nivel estructural y de procedimientos}

Recontrato con los educadores, más allá de las horas de clase.

Espacios físicos y temporales para posibilitar acuerdos y reflexiones conjuntas (creación de talleres, tutorías, espacios para investigación).

Coordinador por equipo.

Coordinación interdisciplinaria.

Compartir en el aula la enseñanza conjunta.

Trabajo conjunto de los educadores de un mismo curso, para posibilitar la mejora en aquellos alumnos con dificultades de aprendizaje.

Canales de comunicación formales y efectivos.

Evaluación del trabajo en equipo: en cada uno de ellos y también en forma global.

\subsection{Propuesta diagnóstica para los equipos}

Cuadro diagnóstico para identificar dónde se producen los problemas, los objetivos que no se cumplen, e impulsar al equipo para planificar las soluciones.

\section{Actitud diagnóstica para mantener la claridad continua}

\begin{tabular}{|l|l|l|l|}
\hline PROBLEMA & $\begin{array}{l}\text { DÓNDE ESTAMOS } \\
\text { AHORA }\end{array}$ & $\begin{array}{l}\text { DÓNDE QUEREMOS } \\
\text { ESTAR DENTRO } \\
\text { DE UN AÑO } \\
\text { (Escala 1-10) }\end{array}$ & $\begin{array}{l}\text { PLAN DE ACCIÓN } \\
\text { ¿QUÉ HAREMOS } \\
\text { PARA LLEGAR? }\end{array}$ \\
\hline
\end{tabular}

Poniendo un fuerte énfasis en lo pedagógico y didáctico, lo que creemos redundará en una mejora de la calidad de la enseñanza. 
Todo esto implica un cambio, y los cambios en ocasiones son dolorosos, a pesar de que se compartan y hasta se haya sido partícipe en su definición. Cambiar supone un concepto simple, pero demanda voluntad, trabajo y disposición, requiere que desaprendamos muchos hábitos, presupuestos, políticas y procedimientos del pasado. Sabemos que los cambios para alcanzar éxito, deben ser graduales, no se puede hacer todo junto, hay que planificarlo desde la institución y por tanto priorizar opciones o decisiones, en la búsqueda de la transformación. Compartimos lo expresado por Frigerio (1997) en relación con que "la institución debe aprender a transitar el camino del cambio, y corresponde a la conducción institucional modelar este proceso a través de la gradualidad de las decisiones que toma"12.

Es nuestra convicción, y no solo nuestro deseo. Aspiramos a que la investigación que realizamos, sea un aporte verdaderamente significativo, para llevar adelante los procesos de cambio que contribuyan a guiar al trabajo en equipo: de la teoría a la cultura institucional.

\section{Notas:}

${ }^{1}$ Gore, E. y Dunlap, D. 1988. Aprendizaje y Organización. Una lectura educativa de las Teorías de la Organización. Buenos Aires: Tesis.

${ }^{2}$ Jares, X. Setiembre-Diciembre 1997. "El lugar del conflicto en la organización escolar". En Revista Iberoamericana de Educación $\mathrm{N}^{\mathrm{o}}$ 15. Micropolítica en la Escuela.

${ }^{3}$ Beltrán Llavador, F. 1996. "Tradición y Cambio en la Dirección Escolar". En Frigerio, Graciela y otros. El análisis de la institución educativa. Hilos para tejer proyectos. Buenos Aires: Santillana.

${ }^{4}$ Fullan, M. y Hargreaves, A. 1999. La escuela que queremos. p.108.

${ }^{5}$ Fullan, M. y Hargreaves, A. 1999. op cit. p.71.

${ }^{6}$ Marchesi, A. y Martín, E. 1998. Calidad de la enseñanza en tiempos de cambio. p. 133.

${ }^{7}$ Fullan, M. y Hargreaves A. 1999. op. cit. p.86.

${ }^{8}$ Taylor, S.J. y Bogdan, R. 1987. Introducción a los métodos cualitativos de investigación. p. 20.

${ }^{9}$ Fernández, L. 1998. El análisis de lo institucional en la escuela. p.52.

${ }^{10}$ Frigerio, G. et al. 1997. Las instituciones educativas. Cara y Ceca. p.69.

${ }^{11}$ Blanchet, A. et al. 1989. Técnicas de investigación en Ciencias Sociales. p.32. * Entrevistado = E

${ }^{12}$ Frigerio, G. et al. 1997. Las instituciones educativas. Cara y Ceca. p.16. 


\section{Bibliografía}

ANTÚNEZ, S. 1998. Claves para la organización de Centros Educativos. $4^{\text {a }}$ edición. Barcelona: Horsori.

BLANCHET, A. et al. 1989. Técnicas de investigación en Ciencias Sociales. Madrid: Narcea.

FERNÁNDEZ, L. 1998. El análisis de lo institucional en la escuela. $1^{a}$ edición. Buenos Aires: Paidós.

FRIGERIO, G. et al. 1997. Las instituciones educativas. Cara y Ceca. $7^{\mathrm{a}}$ edición. Buenos Aires: Troquel.

FULLAN, M. y HARGREAVES, A. 1999. La escuela que queremos. Buenos Aires: Amorrortu.

GAIRÍN, J. Y DARDER VIDAL, P. 1994. Organización de Centros Educativos: aspectos básicos. Barcelona: Praxis.

HARGREAVES, A. 1999. Profesorado, cultura y postmodernidad . $3^{\text {a }}$ edición Madrid: Morata.

MARCHESI, A. y MARTÍN, E. 1998.Calidad de la enseñanza en tiempos de cambio. Madrid: Alianza Editorial.

ROBBINS, H. y FINLEY, M. 1999. Por qué fallan los equipos. Barcelona: Granica.

TAYLOR, S.J. Y BOGDAN, R. 1987. Introducción a los métodos cualitativos de investigación. Buenos Aires: Paidós.

*, ** Diploma en Educación, Universidad ORT Uruguay. 